\title{
A influência do desjejum e do exercício físico na cognição de escolares: uma revisão
}

\author{
The influence of breakfast and physical exercise on \\ cognition in schoolchildren: a review
}

\author{
La influencia del desayuno y ejercicio físico en la cognición \\ de los niños: una revisión
}

\author{
Gustavo Lira Guedes ${ }^{1}$, Jéssica Mycaelle da Silva Barbosa², Claudia \\ Dias Leit ${ }^{3}$, Raiane Maiara dos Santos Pereira ${ }^{4}$, Carmen Sílvia Grubert \\ Campbell ${ }^{5}$, Isabela Almeida Ramos ${ }^{6}$
}

1.Professor de Educação Física. Mestrando do Programa de Pós-Graduação em Educação Física da Universidade Católica de Brasília - PPGEF - UCB, Brasília-DF, Brasil. https://orcid.org/00000001-5259-0098

2.Nutricionista. Egressa do curso de nutrição da Universidade Católica de Brasília - UCB, Brasília-DF, Brasil. https://orcid.org/0000-0002-5421-9732

3.Fisioterapeuta. Doutoranda do Programa de Pós-Graduação em Educação Física da Universidade Católica de Brasília - PPGEF - UCB, Brasília-DF, Brasil. https://orcid.org/00000002-8062-7916

4.Professora de Educação Física, Doutora. Pós-doutoranda do Programa de Pós-Graduação em Educação Física da Universidade Católica de Brasília - PPGEF - UCB, Brasília-DF, Brasil. https://orcid.org/0000-0001-9483-2518

5.Professora de Educação Física, Doutora. Docente do Programa de Pós-Graduação em Educação Física da Universidade Católica de Brasília - PPGEF - UCB, Brasília-DF, Brasil. https://orcid.org/0000-0001-5946-7180

6.Professora de Educação Física, Doutora. Docente do Programa de Pós-Graduação em Educação Física da Universidade Católica de Brasília - PPGEF - UCB, Brasília-DF, Brasil. https://orcid.org/0000-0003-3651-9966

\footnotetext{
Resumo

Introdução. A má alimentação e o sedentarismo estão entre os fatores que mais contribuem para o agravo do estado de saúde na infância, sendo associados a dificuldades e problemas de aprendizagem durante o período de escolarização. Estudos sugerem que crianças em idade escolar podem ter desempenho cognitivo aprimorado pelo consumo regular do café da manhã, bom condicionamento físico e da prática de exercícios físicos. Objetivo. Partindo do princípio de que muitas crianças em idade escolar não consomem o café da manhã todos os dias e, também não atingem as metas de exercício recomendadas por diretrizes de saúde, no presente estudo realizou-se uma revisão narrativa da literatura acerca do consumo do café da manhã, do envolvimento em práticas corporais de crianças, e a sua capacidade de manter e aprimorar as funções cognitivas na infância. Método. A investigação englobou aspectos de consumo, resposta glicêmica e a comparação de carboidratos alimentares presentes no café da manhã, além disso, o envolvimento em aulas de educação física, intervalos ativos e programas de exercício físico e a sua relação com a cognição de crianças em idade escolar. Conclusão. Após revisão de artigos e as suas respectivas contribuições científicas para as temáticas, assumese uma associação positiva entre o consumo adequado do café da manhã e das práticas de exercício físico com o funcionamento cognitivo ideal para crianças em idade escolar. Sendo sugerido pelos autores pesquisados, a adoção e o incentivo dessas práticas para a rotina das crianças durante o período de escolarização.
}

Unitermos. Crianças; desjejum; exercício físico; função executiva 


\begin{abstract}
Introduction. Sedentarism and bad eating habits leading to an increasing risk for diseases in childhood, associated with learning problems during schooling. Studies suggest school children could increase cognitive development thru regularly breakfast consumption, great physical fitness, and physical exercise routine. Objective. If many schoolchildren do not eat breakfast every day and do not attend the exercise guidelines recommendation, the present study performed a narrative review of the literature on breakfast consumption, children's involvement in physical practices, and the ability to maintain and improve cognitive functions in childhood. Method. The investigation comprised aspects of consumption, glycemic response, and the comparison of dietary carbohydrates present at breakfast, besides that, the involvement in physical education classes, active breaks, and exercise programs, and their relationship between cognition and academic achievement of school children. Conclusion. The review indicated that studies and their scientific contribution, it is assumed a positive association among breakfast consumption and physical exercise accordingly guidelines with great cognitive performance in schoolchildren. As suggested by the authors, the adoption and encouragement of these practices for the children's routine during schooling.
\end{abstract}

Keywords. Child; breakfast; exercise; cognition

\begin{abstract}
Resumen
Introdución. La mala alimentación y la inactividad física se encuentran entre los factores que más contribuyen al deterioro del estado de salud en la infancia, estando asociados a dificultades de aprendizaje y problemas durante la escolarización. Los estudios sugieren que los niños en edad escolar pueden haber mejorado su rendimiento cognitivo a través del desayuno regular, un buen acondicionamiento físico y ejercicio físico. Objetivo. Suponiendo que muchos niños en edad escolar no desayunan todos los días y tampoco alcanzan las metas de ejercicio recomendadas por las guías de salud, en el presente estudio se realizó una revisión narrativa de la literatura sobre el consumo de desayuno, participación en las prácticas corporales de los niños y su capacidad para mantener y mejorar las funciones cognitivas en la infancia. Método. La investigación abarcó aspectos de consumo, respuesta glucémica y la comparación de carbohidratos dietéticos presentes en el desayuno, además de la participación en clases de educación física, descansos activos y programas de ejercicio y su relación con la cognición de los niños pequeños. Conclusión. Después de revisar los artículos y sus respectivos aportes científicos a los temas, se asume una asociación positiva entre el consumo adecuado de desayunos y las prácticas de ejercicio físico con el funcionamiento cognitivo ideal para los niños en edad escolar. Como sugieren los autores investigados, la adopción y fomento de estas prácticas para la rutina de los niños durante el período escolar.
\end{abstract}

Palabras clave. Niño; desayuno; ejercicio físico; función ejecutiva

Trabalho realizado na Universidade Católica de Brasília, Programa de Pós-Graduação em Educação Física, Laboratório de Estudos em Educação Física e Saúde (LEEFS). Brasília-DF, Brasil.

\title{
INTRODUÇÃO
}

Ao longo de algumas décadas, desde o incremento impactante dos avanços tecnológicos, o estilo de vida das crianças tem mudado drasticamente, de brincadeiras e jogos com exigência física e dietas predominantemente naturais 
para rotinas fisicamente inativas, baixa adesão ao consumo do café da manhã (CM) e aumento da ingestão de alimentos com baixa qualidade nutricional de alimentos industrializados ${ }^{1-3}$. Embora existam muitas lacunas no conhecimento atual sobre como superar o sedentarismo e a má alimentação, a condição física e o estado nutricional de uma criança possuem papel fundamental para 0 desenvolvimento de estruturas e funções cerebrais, desempenho escolar e saúde cognitiva ${ }^{4-7}$.

Os comportamentos relacionados à saúde durante a infância desempenham um importante papel para habilidades mentais específicas, o que tem sido cada vez mais evidenciado em pesquisas científicas. As funções executivas ou funções cognitivas são um conjunto de habilidades que podem ser influenciadas positivamente pelos comportamentos relacionados à saúde, e estão envolvidas na capacidade de inibição atencional (resistir a distrações e direcionar o foco), reter e recuperar informações (memória de trabalho), raciocínio, flexibilidade de tarefas, tomada de decisão, planejamento, resolução de problemas e atenção ${ }^{8}$. Estudos realizados com a aquisição de imagens e funções cerebrais estão revelando a aparência de um cérebro saudável e ajudando os pesquisadores a entender os mecanismos neurais responsáveis pelo aprimoramento do controle cognitivo e por consequência um melhor aprendizado5,9,10. A partir dessa perspectiva, muitos comportamentos são capazes de impactar e contribuir substancialmente para as funções cognitivas, sendo 
constantemente documentado como importante indicador para a saúde física e mental, e para o sucesso na escola e na vida.

O consumo do CM tem sido considerado um dos hábitos capazes de impactar as funções executivas de crianças, sendo recomendado como parte de uma dieta benéfica. Os consumidores regulares de CM estão propensos a ter uma melhor ingestão de nutrientes, peso corporal e estilo de vida mais saudáveis, além de desempenho acadêmico e funções cognitivas aprimorados ${ }^{11-15}$. Pesquisas sobre a influência da composição do CM e de sua ausência para os processos mentais de curto prazo podem fornecer orientações efetivas a respeito dessa temática, devido ao efeito potencial da qualidade da dieta em atenuar o declínio energético e cognitivo nas primeiras horas do dia ${ }^{16-18}$.

Por sua vez, o exercício físico (EF) é amplamente recomendado para a obtenção de benefícios em marcadores de saúde e aprimoramento funcional cognitivo e saúde cerebral na infância17,19. Muitos mecanismos foram propostos para explicar as mudanças no desempenho cognitivo após o EF, incluindo a excitação geral do córtex cerebral $^{20}$, aumentos no fluxo sanguíneo cerebral ${ }^{21}$ e a regulação positiva da atividade neuronal ${ }^{22}$. De toda forma, criar um ambiente em modelo laboratorial, que seja atingível no mundo real com base em descobertas empíricas na literatura cognitiva resultou em uma ampla variedade de protocolos que diferem em tipo de exercício, intensidade e duração 23,24 . 
O CM e o EF estão intrinsecamente ligados a saúde do nosso cérebro, eles foram entrelaçados durante nosso passado evolutivo, bem como em nosso presente cultural e biológico. Considerando que esses hábitos têm uma forte relação com o favorecimento de funções cognitivas durante a infância, pretende-se realizar de modo reflexivo um levantamento das evidências da literatura científica sobre os efeitos nutricionais do $\mathrm{CM}$ e de atividades motoras no desempenho cognitivo de crianças em idade escolar. Tratase de uma observação de descobertas empíricas, fundamentadas em torno de uma cultura que estimule a prática de EF e do consumo do CM para crianças, na tentativa de circunstanciar as temáticas elencando a influência positiva e negativa quando essas práticas são acatadas ou negligenciadas, considerando a necessidade de modificar as políticas escolares e a criação de ambientes que incentivem esses hábitos na escola e na rotina familiar.

\section{MÉTODO}

Visando o objetivo apontado, optou-se pela revisão narrativa, por proporcionar uma síntese do conhecimento atual nos temas delimitados, em numerosas fontes de informações publicadas anteriormente. Além disso, esse método de revisão da literatura relata as descobertas do autor em um formato condensado que normalmente resume o conteúdo de cada artigo, possibilita uma discussão ampliada em virtude da abrangência dos temas levantados ${ }^{25}$. 
O levantamento bibliográfico foi realizado no período de outubro de 2019 a outubro de 2020 nas bases de dados SciELO e PubMed, assim como o Google Scholar, por serem ferramentas simples e poderosas com uma vasta coleção de publicações no âmbito da saúde, abrangendo as publicações entre os anos de 2005 a 2020, nos idiomas Português e Inglês. O presente estudo não teve como característica metodológica a análise quantitativa, não expressando numericamente os artigos originais e de revisão atenderam os critérios originais e que foram selecionados, revisados e posteriormente integrados ao texto.

As buscas se basearam na pergunta de pesquisa: Como aprimorar e sustentar o desempenho de funções cognitivas em crianças, considerando aspectos nutricionais do desjejum e da prática de exercícios físicos? As buscas foram realizadas em duas etapas descritas em sequência, compostas pelas palavras-chave: $1^{\circ}$ - Breakfast ou skipping breakfast ou Glycaemic index ou glycaemic load ou Glucose metabolism; $2^{\circ}$ - Physical exercise ou physical activity ou Acute exercise ou physical exercise program ou Aerobic fitness ou physically active. As duas etapas foram realizadas em pesquisa avançada com os operadores booleanos que combinaram (AND) com: (Cognition ou executive functions) AND (Children ou pre-adolescents).

Atendendo as temáticas e processos de trabalhos discorridos nos artigos analisados, os estudos foram agrupados e distribuídos em duas grandes categorias, que discutem a importância do CM e do EF para a saúde cognitiva 
e por consequência para o aprendizado de crianças em idade escolar.

\section{RESULTADOS e DISCUSSÃO}

\section{Café da manhã, resposta glicêmica e cognição}

O CM é considerado a mais importante refeição do dia sendo recomendado como parte de um plano alimentar saudável para crianças em idade escolar. Apesar de muitos benefícios do seu consumo regular, de acordo com a literatura, muitas crianças não consomem esta refeição todos os dias ${ }^{15,26-28}$. Em uma revisão de Rampersaud ${ }^{26}$ foi demonstrado que as crianças que costumam pular o CM estão na pré-adolescência, e os motivos mais citados incluem não ter tempo suficiente pela manhã para comer, não sentir fome ou não sentir vontade de comer e preferir dormir até a hora de ir para a escola.

Um padrão regular de refeições, consumindo uma porção maior de energia no início do dia está associado a diversos benefícios fisiológicos, principalmente para o metabolismo da glicose que desempenha um papel central em todos os processos celulares, incluindo nas células nervosas, sendo a principal fonte de energia disponível para o funcionamento adequado de todo tecido cerebral ${ }^{29,30}$.

As demandas de energia do cérebro são altas: o consumo frequentemente citado é de $20 \%$ do gasto energético diário do corpo, apesar de ser responsável por apenas $2 \%$ da massa corporal total ${ }^{31}$. Contudo, a necessidade metabólica de glicose é muito superior durante 
a infância, alcançando uma taxa equivalente a $43 \%$ da necessidade diária de energia do corpo $^{32}$. Além disso, as crianças apresentam um estresse metabólico superior aos adultos durante o período do sono, consumindo uma maior quantidade de glicose dos estoques de glicogênio ${ }^{33}$. Nesse sentido, a ausência do CM na rotina das crianças pode provocar maiores concentrações de insulina pós-prandial, sugerindo uma inflexibilidade metabólica que pode ser prejudicial à homeostase glicêmica ${ }^{34-36}$. A regulação da glicose sanguínea pode ser um mecanismo responsável por alterar o desempenho em muitos domínios cognitivos, principalmente contra o seu declínio nas primeiras horas do dia, podendo facilitar a motivação, a concentração e a retenção de informações, processos cognitivos estes de fundamental importância para a realização de tarefas escolares ${ }^{4,16,36}$.

A ingestão diária do CM possui relação positiva com um crescimento e desenvolvimento ideal na infância, incluindo o potencial aprimoramento cognitivo e acadêmico que a ciência tem procurado diligentemente consolidar ${ }^{13,14,30,37 .}$ Diferenças abrangentes no desempenho cognitivo entre crianças que consumiram o $\mathrm{CM}$ e aqueles que não consumiram foram observadas em um estudo com 1386 escolares com média de idade de 11 anos. A capacidade de evitar a distração, focar a atenção e de se manter atento foi comprometida em $7 \%$ das crianças que permaneceram em jejum, além de um declínio na velocidade e acurácia das respostas em comparação ao grupo que consumiu o $\mathrm{CM}^{38}$. 
A infância é caracterizada pela aquisição e desempenho em diversos processos cognitivos, e o consumo frequente e adequado do $\mathrm{CM}$ pode contribuir com a otimização da alta taxa de utilização energética decorrente do intenso aprendizado nessa fase da vida. Consequentemente, tem havido um interesse crescente nos efeitos da qualidade nutricional no desempenho cognitivo de crianças e, mais especificamente, em como o desempenho cognitivo pode ser aperfeiçoado através de intervenções nutricionais.

A ciência da nutrição estuda a relação entre os alimentos e nutrientes ingeridos e seu equilíbrio associados à saúde ${ }^{1,39,40}$. Dentre os ingredientes principais dos alimentos, os carboidratos têm sido amplamente utilizados em investigações que avaliam a sua formulação (qualidade e quantidade) e sua capacidade de influenciar aspectos seletivos do processamento cognitivo de crianças ${ }^{4,41}$. O índice glicêmico (IG) de um alimento contendo carboidratos representa a rapidez com que a glicose sanguínea aumenta após sua ingestão, podendo ser caracterizado em escala numérica como IG baixo, moderado ou alto, fornecendo assim uma medida da qualidade e da taxa de absorção dos carboidratos do alimento ${ }^{42}$.

Para elucidar a relação entre a qualidade dos carboidratos no CM e a cognição em crianças de 6 a 11 anos, Ingwersen et $a l^{43}$ observaram que o desempenho cognitivo dessas crianças sofreu uma redução ao longo da manhã, e que esse declínio pode ser significativamente reduzido após a ingestão de um CM de baixo IG quando comparado ao de 
alto IG. Neste estudo foi observado resultados significantemente superiores na acurácia em teste de atenção, e na retenção e recuperação das informações em um teste de avaliação da memória quando o CM foi de baixo IG. Outros estudos ressaltam que a composição de alimentos de baixo IG pode fornecer uma fonte de energia mais lenta e sustentada, com uma atenuação significante do declínio cognitivo pela manhã, resultado esse importante para uma devida recomendação de um CM mais adequado para as crianças antes de irem para a escola ${ }^{18,44}$.

Paralelamente a esses achados, estudos documentaram a importância da distinção das respostas glicêmicas levando em consideração o IG e a carga glicêmica (CG) ${ }^{45,46}$. A CG é o produto do IG do alimento pela quantidade de carboidrato contida no alimento consumido, sendo este considerado o preditor mais robusto da resposta glicêmica ${ }^{45}$. Em termos de recomendações nutricionais, o CM com baixa CG minimiza as oscilações nas concentrações de glicose sanguínea e pode ser benéfico para o desempenho cognitivo durante a manhã $16,47,48$.

Benton et $a / 47$ investigaram durante 4 semanas 0 impacto da ingestão de refeições com diferentes CG, porém com semelhantes calorias sobre o desempenho da memória e a atenção de crianças de 6 a 7 anos. As crianças foram avaliadas individualmente no intervalo de duas a três horas após o CM ser consumido, e foi observada melhor recordação imediata em teste de memória verbal e maior capacidade de manter a atenção após o consumo do CM de baixa CG, além 
de uma menor interrupção com distrações em tarefas típicas como escrever e fazer cálculos nesse grupo quando comparado ao grupo CM de moderada e de alta CG.

A alimentação inadequada em quantidade e qualidade tem prejudicado a saúde das crianças, elevando a incidência de doenças como a obesidade e a diabetes que estão adquirindo proporções epidêmicas na população pediátrica ${ }^{12,49,50}$. Evidências atuais da relação intestinocérebro indicam que, aliado à dieta, certas bactérias intestinais influenciam o metabolismo e a absorção de nutrientes, auxiliando na digestão de carboidratos e na síntese de compostos bioativos ${ }^{51,52}$. O intestino e todos os seus residentes microbianos, portanto, influenciam diretamente aspectos da imunidade, função endócrina e o sistema nervoso, desempenhando um papel extremamente importante para a saúde humana, atuando na proteção ou tratamento de doenças e de interesse particular para essa revisão no metabolismo de alimentos ${ }^{53}$.

Mais uma vez a qualidade do carboidrato se mostra de extrema importância para a saúde humana, as espécies bacterianas benéficas de que precisamos em nosso sistema digestivo podem ser mantidas por uma dieta rica em fibras, particularmente por carboidratos acessíveis à microbiota, que são encontrados principalmente nos em alimentos in natura ${ }^{51}$. Por sua vez, o aumento da ingestão desses alimentos que são fonte de fibras alimentares melhora o controle glicêmico e a sensibilidade a insulina ${ }^{39,54}$, processos 
fisiológicos cogitados para a melhoria da saúde física e cognitiva.

Em um estudo de delineamento cruzado, 30 crianças com idades entre 6 e 11 anos completaram uma bateria de testes cognitivos durante 3 semanas, após o consumo de um CM de baixo IG e alto teor de fibras (aveia), um CM com baixo teor de fibra e alto IG (cereal matinal) ou sem CM. Os resultados foram comparados pelo sexo e pela faixa etária (6-8 anos crianças mais jovens) e (9-11 anos préadolescentes). A ingestão do CM com alto teor de fibra e baixo IG apresentou melhorias no desempenho cognitivo em tarefas que requerem processamento de uma exibição visual complexa em pré-adolescentes, já as crianças mais jovens apresentaram memória espacial e atenção auditiva aprimorada, enquanto as meninas dessa mesma faixa de idade apresentaram um melhor desempenho no teste de memória de curto prazo ${ }^{18}$.

No estudo de Khan et al ${ }^{17}$ foram avaliados a composição alimentar e a inibição atencional (Flanker Task) de crianças de 7 a 9 anos de idade participantes de um programa pósescola com duração de 9 meses de atividades físicas Fitness Improving Thinking (FITKIDS). Os principais achados foram que uma ingestão diária mais elevada de fibras alimentares totais e insolúveis foi positivamente associada com a precisão em todas as etapas de uma tarefa de inibição atencional, enquanto a ingestão fibras solúveis foi seletivamente associada a um maior desempenho nos ensaios mais difíceis do teste. Além disso, um dos pontos 
fortes do presente estudo incluíram o ajuste de covariáveis conhecidas por influenciar a função cognitiva infantil, em especial o condicionamento físico dos participantes.

Um declínio no consumo do CM tem uma relação direta com o condicionamento físico, aumentando a probabilidade de as crianças tornarem-se fisicamente inativas e ter um baixo nível de aptidão cardiorrespiratória ${ }^{55}$. Evidências convergentes indicam que a prática de EF e o condicionamento físico são correlatos-chave da função cognitiva infantil, permitindo a comunicação cruzada entre os músculos esqueléticos e o cérebro, provocando uma variedade de adaptações homeostáticas celulares, incluindo o aumento da captação de glicose devido às demandas metabólicas e mecânicas ${ }^{5,56}$.

\section{Aptidão física, práticas motoras e cognição}

O ditado popular "o que é bom para o coração é bom para o cérebro" exprime descobertas científicas referentes ao efeito benéfico do EF nas funções cognitivas, melhorandoas por toda a vida, e protegendo do declínio pertinente à senescência ${ }^{24}$. O exercício representa um grande desafio para a homeostase de várias células, tecidos e órgãos do corpo em resposta a um aumento da atividade metabólica dos músculos esqueléticos em contração. Além disso, é capaz de aumentar o fluxo sanguíneo para o cérebro sendo que: a) Aumenta a secreção neuroquímica de metabólitos que fornecem energia ao cérebro e diminui as que sinalizam estresse emocional e prejuízo cognitivo; b) Aumenta a 
secreção de neurotrofinas que protegem os neurônios existentes e estimulam o crescimento da vascularização e a produção de novos tecidos cerebrais; e c) Intercede na liberação de neurotransmissores excitatórios, inibitórios e os que cumprem os dois papéis, otimizando a comunicação nas regiões cerebrais importantes para a saúde funcional do cérebro ${ }^{24}$.

Existem muitos fatores que influenciam a saúde física e mental durante infância e é onde a cinesiologia e a neurociência cognitiva se completam. Está bem estabelecido que o EF tem implicações consideráveis no desempenho de uma série de capacidades que regulam, controlam e planejam o comportamento e os processos cognitivos incluindo o controle inibitório ${ }^{57}$, atenção seletiva ${ }^{58}$, memória de trabalho $^{59}$ e flexibilidade cognitiva ${ }^{60}$, habilidades essenciais para o sucesso na escola61.

No entanto, aprofundando-se em questões sobre esse engajamento benéfico para crianças, percebe-se na atualidade uma infância acompanhada de exigências e horários rígidos, com uma jornada escolar onde são dedicadas mais da metade das horas de vigília diária e é normalmente endereçado a atividades de cunho sedentário ${ }^{62,63}$. Além disso, a maior ênfase nas avaliações de ensino e o discurso equivocado sobre a pouca utilidade da educação física escolar, agrava ainda mais essa realidade ao incentivar as escolas a eliminarem oportunidades de EF durante o dia escolar, incluindo o recreio e as aulas de educação física64. Períodos prolongados de instrução 
acadêmica hipocinéticas podem ocasionar um estado de impaciência ou inquietude reduzindo a concentração e sendo prejudicial a experiência do aprendizado65.

Considerando que grande parte da rotina da criança é na escola, intervenções nesse ambiente podem desempenhar um papel importante na prevenção de hábitos sedentários, criando um ambiente saudável que promova atividade física durante todo o dia escolar para os alunos, e os auxiliem a cumprir os 60 minutos recomendados de EF moderado a vigoroso diário66,67. Em tese, as crianças aumentam seu nível de concentração e comportamento em sala de aula e no desempenho de tarefas, após a participação em EF por meio do recreio $^{68}$, aulas de educação física69, pausa ativa ${ }^{70-72}$, e reforço escolar ativo ${ }^{73}$, além disso, benefícios adicionais são observados em leitura, escrita e aritmética $^{74-80}$.

Sendo assim, muitas pesquisas foram desenvolvidas com EF em consonância com as missões acadêmicas escolares. Em um programa longitudinal, com duração de 3 anos, comparou-se as pontuações acadêmicas de alunos que realizavam cinco aulas semanais de Educação Física com alunos que permaneceram na programação da escola de duas aulas por semana. Os autores demonstraram que 0 incremento das aulas de Educação Física na rotina escolar pode ser útil como instrumento pedagógico, com resultados positivos na capacidade de leitura, escrita e na pontuação da disciplina de matemática em crianças que iniciaram a pesquisa aos 6 anos de idade ${ }^{74}$. Reflexão semelhante pode- 
se extrair de outro estudo com 3 anos de intervalo ativo durante as aulas que contou com a participação de 24 escolas, apenas 90 minutos semanais de EF de intensidade moderada a vigorosa nomeado Physical Activity Across the Curriculum (PAAC), foram capazes de melhorar 0 desempenho geral de crianças em um teste acadêmico padronizado em $6 \%$, enquanto o envolvimento nas aulas sem as pausas ativas melhorou $1 \%{ }^{61}$.

Tais descobertas reforçam que o EF pode contribuir para o processo de ensino-aprendizagem e, oferecendo aos alunos uma maior oportunidade de se envolver em comportamentos saudáveis, além de efeitos positivos no desempenho acadêmico $61,72,75,76$. É importante ressaltar que essas melhorias no desempenho acadêmico de crianças se fortalecem examinando resultados comportamentais em tarefas cognitivas laboratoriais ${ }^{5}$. Além disso, levando em consideração que as funções cognitivas podem ser sensíveis ao $\mathrm{EF}^{77}$, muitos estudos concentraram seus esforços em examinar os efeitos agudos e crônicos nos processos cognitivos de controle executivo, devido ao fato desses processos concederem a base para uma aprendizagem bemsucedida e desempenho escolar de crianças $^{23,69,76,78,79-82 . ~}$

Um campo cada vez mais robusto da literatura neurocientífica examina a influência de uma única sessão de EF, no comportamento cognitivo de crianças, mensurando principalmente componentes de aspectos atencionais e percepcionais. Drollette et $a / 83$, realizaram um estudo com exercício agudo de intensidade moderada em dois grupos de 
pré-adolescentes com alta e baixa performance em teste que avaliou o controle inibitório atencional (Flanker Modificado). Os pré-adolescentes que apresentaram baixa performance cognitiva apresentaram melhores resultados no mesmo teste após $20 \mathrm{~min}$ de caminhada em esteira. Além disso, os índices neuroelétricos responsáveis por uma melhor alocação atencional foram observados em ambos os grupos após 0 término do exercício. Sendo assim, os resultados apresentados pelo referido estudo sugerem que sessões de exercício de intensidade moderada podem ter maiores benefícios para as crianças caracterizadas pela capacidade de controle inibitório atencional inferior.

Em outra intervenção aguda, Altenburg et a/84, compararam os efeitos de nenhuma, uma ou duas sessões de 20 minutos de EF em atividade de dança em intensidade moderada durante a manhã escolar sobre a atenção seletiva de crianças de 10 a 13 anos. O teste de atenção seletiva foi reproduzido durante todo o período matutino $\left(0^{\prime} ; 20^{\prime} ; 110^{\prime}\right.$; $220^{\prime}$ minutos), quando comparados os resultados, o grupo de duas sessões de EF apresentou o resultado mais expressivo levando em consideração a média de todos os testes de atenção seletiva reproduzidos durante a manhã escolar. Outro estudo interdisciplinar com EF agudo que contou com a participação de 35 crianças com idade entre 9 e 11 anos, o efeito de 10 minutos EF aeróbio integrada à disciplina de matemática melhorou o tempo de resposta das crianças em uma tarefa de controle inibitório atencional, em comparação à prática da disciplina quando permaneciam 
sentados. Além disso, as crianças categorizadas com sobrepeso apresentaram também uma melhor acurácia, sendo observados benefícios superiores a esse grupo no teste de controle inibitório após a prática de 10' de EF aeróbio ${ }^{85}$.

Desse modo, proporcionar pausas ativas e também combinar conteúdos escolares aos EF podem impactar positivamente a capacidade de seleção e inibição atencional que desempenham um papel crítico na prevenção de comportamentos indesejados, uma vez que se relacionam com a capacidade de agir com base na escolha e não no impulso, processos centrais para manter a atenção e o controle sobre as próprias ações ${ }^{86,87}$.

Considerando outros fatores, como o envolvimento em programas de atividade física de longo prazo (EF crônico), estudos destacam melhorias que vão além das observadas nas variáveis cognitivas, mas também no aumento da aptidão aeróbia dos participantes. Scudder et $a / 88$, investigaram se as mudanças na aptidão aeróbia estão associadas a modulações no controle inibitório e memória de trabalho de crianças. Foram incluídas duas sessões de 10 minutos de lições acadêmicas fisicamente ativa durante o dia escolar, aproximadamente 3 anos depois, níveis mais altos de aptidão física foram associados a melhora no desempenho geral de cada tarefa (controle inibitório e memória de trabalho), especialmente para as condições mais difíceis. A importância da memória de trabalho para a cognição infantil é esclarecida pela capacidade de reter, manipular as 
informações e pelo seu notável poder de influenciar outras habilidades cognitivas complexas, como a flexibilidade cognitiva que envolve a visualização mental sobre algo de diferentes perspectivas, podendo solucionar problemas e conceber ideias que não haviam sido consideradas antes ${ }^{8}$.

Em um programa pós-escola com intervenção crônica, foi ofertado 70 minutos de atividade física moderada a vigorosa por dia da semana durante 9 meses, as crianças eram selecionadas aleatoriamente para uma lista de espera ou participar do Fitness Improving Thinking (FITKids). Além de apresentarem melhoras na aptidão aeróbica, crianças que participaram do FITKids obtiveram melhorias em medidas comportamentais de alocação atencional (Inibição e Flexibilidade cognitiva), quando comparadas ao pré e pósteste e com as crianças que permaneceram na lista de espera ${ }^{61}$. Nesse sentido, vários relatórios com crianças de condicionamento físico alto foram publicados a partir do ensaio FITKids, contribuindo de forma robusta para a literatura neurocientífica que recomenda a adoção de um estilo de vida mais promissor no que se refere à saúde global $10,59,60,89$.

Apesar dos índices neuroelétricos e métodos de neuroimagem não estarem integrados aos objetivos desta revisão, estudos com essas técnicas têm demonstrado que crianças com um alto condicionamento físico apresentam um maior volume de estruturas cerebrais, além de uma promoção mais eficiente de atividade e conectividade cerebral $^{90,91}$. De interesse específico para esta revisão, linhas 
convergentes de pesquisa indicam que uma aptidão física aprimorada na infância está associada a um desenvolvimento cerebral estrutural e funcional vantajoso ${ }^{17}$.

Em contrapartida, atualmente as crianças enfrentam realidades que limitam as oportunidades de envolvimento em práticas de exercício físico sendo atribuído a um baixo nível de aptidão física, por exemplo, bairros inseguros, falta de espaços para brincar, aumento do tempo de navegação na internet e exposição a diferentes tipos de tela e aumento da exigência por desempenho acadêmico ${ }^{92-95}$. Em nosso levantamento de estudos para a composição dessa temática não encontramos dados empíricos na literatura que sugiram que o aumento do tempo gasto em atividades físicas (Nas aulas de Educação Física, no contraturno escolar e aulas de reforço) apontem para um efeito prejudicial sobre o desempenho acadêmico e cognitivo de crianças. Destacando, assim, a importância de se recomendar os exercícios físicos para a obtenção de benefícios acadêmicos e cognitivos de crianças, devido a extensa literatura que aponta esse relacionamento positivo. O quadro 2 apresenta de forma resumida os resultados dos estudos que foram a base da composição da temática sobre EF e funções executivas de crianças.

\section{CONCLUSÃO}

A partir dos principais resultados levantados pelos estudos analisados elaborou-se a Figura 1, no qual aponta de modo hierárquico o que deve ser evitado e o que deve ser 
consumido no CM para a manutenção e o sustento de habilidades cognitivas durante a manhã escolar para crianças, também ressalta a importância de se evitar comportamentos sedentários e se envolver das práticas de exercício físico, aulas de educação física e ter um bom condicionamento físico para a melhoria da saúde cognitiva dos mesmos.

O cérebro em rápido desenvolvimento de uma criança é sensível às experiências, sendo pautada por uma série de interações ambientais, o envolvimento em comportamentos de saúde promove um forte e benéfico equilíbrio em muitos domínios do universo infantil, sendo amplamente estudados, principalmente por seus potenciais efeitos benéficos no funcionamento cognitivo e por consequência no desempenho acadêmico. Dentre esses comportamentos destacam-se o consumo de alimentos saudáveis, a periodicidade das refeições e os exercícios físicos que são fatores importantes para processos cognitivos.

Com base nas evidências descritas no presente estudo, defendemos quatro princípios abrangentes: 10 - a ingestão do CM é um método eficaz para combater a falta de concentração, que pode ser ocasionada pelo desequilíbrio energético gerado pelo jejum; 20 - apesar de não apresentar resultados robustos e uniformes a qualidade nutricional do desjejum pode ter relação com aspectos cognitivos, 
Figura 1. Representação gráfica de práticas físicas e nutricionais que podem impactar as funções de crianças.

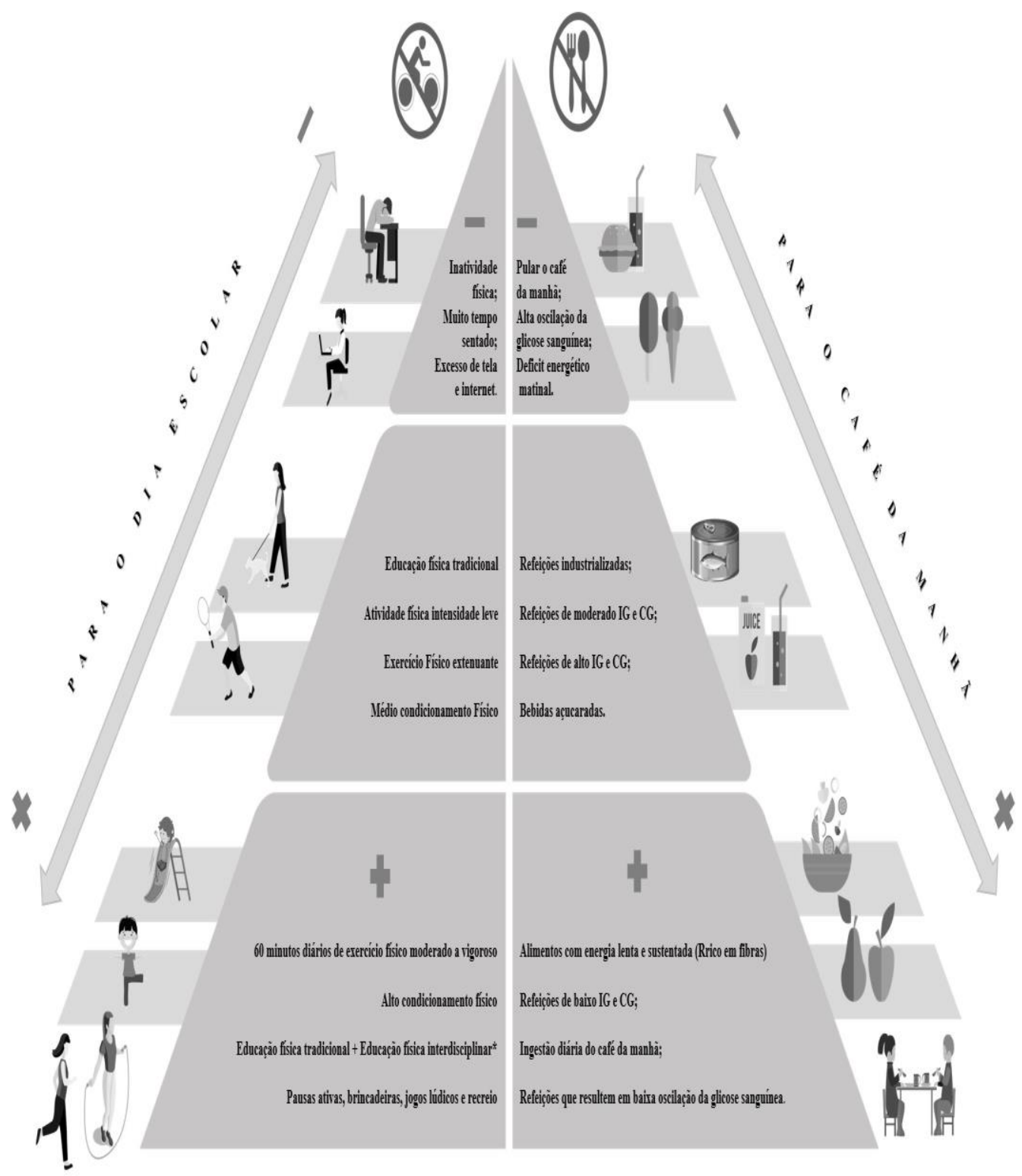

Representação hierárquica de práticas capazes de influenciar as funções cognitivas de crianças. À esquerda recomendações acerca de atividades motoras e à direita do consumo do café da manhã. No topo da pirâmide estão representadas as práticas motoras e nutricionais que não são recomendadas, na faixa central as que devem ser adotadas com cautela, e na base da pirâmide as práticas amplamente recomendadas pela literatura científica e por diretrizes de saúde. *Educação Física interdisciplinar (aulas de educação física integradas à outras disciplinas do currículo escolar). IG - Índice Glicêmico; CG - Carga Glicêmica.

por apresentar resultados significantes na homeostase glicêmica, diminuindo as oscilações energéticas e contribuindo principalmente para a atenuação do declínio 
cognitivo matinal; 30 - aulas de educação física, pausas ativas e recreio contribuem para o processo de aprendizagem e estimulam as crianças a se tornarem mais saudáveis e ativas, impactando positivamente em resultados de testes cognitivos e de forma indireta no desempenho acadêmico; $4^{\circ}$ - crianças com alto condicionamento físico e que se envolvem em programas crônicos de exercício físico apresentam desempenho superior em testes cognitivos em comparação aos seus pares com menor envolvimento e condicionamento físico.

Por fim, uma alimentação com qualidade e exercícios praticados com regularidade são essenciais, tanto para o crescimento como para a manutenção da vida, mas não podemos esquecer que o desequilíbrio de ambas também pode ser responsável pelo acometimento de distúrbios e doenças ${ }^{2,3,38,48,64,65}$. Fato alarmante para as famílias, gestores escolares e as organizações de saúde, uma vez que esse desequilíbrio identificado na infância pode se perpetuar durante toda a vida, formando uma geração de adultos com sérios riscos relacionados à saúde ${ }^{96,97}$. A estimulação de comportamentos saudáveis reduzirá os riscos de problemas de aprendizagem, fazendo com que crianças desfrutem de uma vida mais saudável, saborosa e feliz.

\section{AGRADECIMENTOS}

Os autores agradecem a Coordenação de Aperfeiçoamento Pessoal de Nível Superior (CAPES), pela bolsa de estudos de mestrado concedida ao GLG, também ao 
Fundo de Amparo à Pesquisa do Distrito Federal (FAPDF), pelo aporte financeiro em visita Técnica, que contribuiu para a aproximação das temáticas elencadas.

\section{REFERÊNCIAS}

1.WHO - World Health Organization. Obesity and overweight (endereço na internet). (acessado em 01/042020. Disponível em: https://www.who.int/en/news-room/fact-sheets/detail/obesity-andoverweight\#

2. Malik VS, Willett WC, Hu FB. Global obesity: trends, risk factors and policy implications. Nat Rev Endocrinol 2013;9:13-27. https://doi.org/10.1038/nrendo.2012.199

3. Martins AP, Levy RB, Claro RM, Moubarac JC, Monteiro CA. Increased contribution of ultra-processed food products in the Brazilian diet (1987-2009). Rev Saude Publica 2013;47:656-65. https://doi.org/10.1590/S0034-8910.2013047004968

4. Hoyland A, Dye L, Lawton CL. A systematic review of the effect of breakfast on the cognitive performance of children and adolescents. Nutr Res Rev 2009;22:220-43. https://doi.org/10.1017/S0954422409990175

5.Erickson KI, Hillman $\mathrm{CH}$, Kramer AF. Physical activity, brain, and cognition. Curr Op Behav Sci 2015;4:27-32. https://doi.org/10.1016/j. cobeha.2015.01.005

6. Hillman $\mathrm{CH}$, Khan NA, Kao SC. The Relationship of Health Behaviors to Childhood Cognition and Brain Health. Ann Nutr Metab 2015;66 (Suppl 3):1-4. https://doi.org/10.1159/000381237

7.Kao SC, Drollette ES, Scudder MR, Raine LB, Westfall DR, Pontifex $\mathrm{MB}$, et al. Aerobic Fitness Is Associated With Cognitive Control Strategy in Preadolescent Children. J Mot Behav 2017;49:150-62. https://doi.org/10.1080/00222895.2016.1161594

8. Diamond A. Executive functions. Annu Rev Psychol 2013;64:135-68. https://doi.org/10.1146/annurev-psych-113011-143750

9. Feldman DE. Synaptic mechanisms for plasticity in neocortex. Annu Rev Neurosci

2009;32:33-55.

https://doi.org/10.1146/annurev.neuro.051508.135516

10.Chaddock-Heyman L, Erickson KI, Voss MW, Knecht AM, Pontifex MB, Castelli DM, et al. The effects of physical activity on functional MRI activation associated with cognitive control in children: a randomized controlled intervention. Front Hum Neurosci 2013;7:72. https://doi.org/10.3389/fnhum.2013.00072

11.Ruxton $\mathrm{CH}$, Kirk TR. Breakfast: a review of associations with measures of dietary intake, physiology and biochemistry. $\mathrm{Br} \mathrm{J}$ Nutr 1997;78:199-213. https://doi.org/10.1079/bjn19970140

12. de la Hunty A, Gibson S, Ashwell M. Does regular breakfast cereal consumption help children and adolescents stay slimmer? A systematic review and meta-analysis. Obes Facts 2013;6:70-85. 
https://doi.org/10.1159/000348878

13. Littlecott HJ, Moore GF, Moore L, Lyons RA, Murphy S. 'Breakfast: how important is it really?' A response. Public Health Nutr 2016;19:1720-1. https://doi.org/10.1017/S1368980016000380

14.Rampersaud GC, Pereira MA, Girard BL, Adams J, Metzl JD. Breakfast habits, nutritional status, body weight, and academic performance in children and adolescents. J Am Diet Assoc 2005;105:743-62. https://doi.org/10.1016/j.jada.2005.02.007

15.O"Neil CE, Nicklas TA. Breakfast Consumption versus Breakfast Skipping: The Effect on Nutrient Intake, Weight, and Cognition. Nestle Nutr Inst Workshop Ser 2019;91:153-67. https://doi.org/10.1159/000493707

16.Sünram-Lea SI. Breakfast, Glycemic Index, and Cognitive Function in School Children: Evidence, Methods, and Mechanisms. Nestle Nutr Inst Workshop Ser 2019;91:169-78. https://doi.org/10.1159/000493708

17. Khan NA, Raine LB, Drollette ES, Scudder MR, Kramer AF, Hillman $\mathrm{CH}$. Dietary fiber is positively associated with cognitive control among prepubertal children. J Nutr 2015;145:143-9. https://doi.org/10.3945/in.114.198457

18. Mahoney CR, Taylor HA, Kanarek RB, Samuel P. Effect of breakfast composition on cognitive processes in elementary school children. Physiol Behav 2005; 85:635-45.

https://doi.org/10.1016/j.physbeh.2005.06.023

19. Khan NA, Hillman $\mathrm{CH}$. The relation of childhood physical activity and aerobic fitness to brain function and cognition: a review. Pediatr Exerc Sci 2014;26:138-46. https://doi.org/10.1123/pes.2013-0125 20.Kamijo K, Nishihira Y, Hatta A, Kaneda T, Kida T, Higashiura T, et al. Changes in arousal level by differential exercise intensity. Clin Neurophysiol 2004;115:2693-8. https://doi.org/10.1016/j.clinph.2004.06.016

21. Merege Filho CAA, Alves CRR, Sepúlveda CA, Costa A dos S, Lancha Junior AH, Gualano B. Influência do exercício físico na cognição: uma atualização sobre mecanismos fisiológicos. Rev Bras Med Esp 2014;20:237-41.

http://dx.doi.org/10.1590/151786922014200301930

22. Hillman $\mathrm{CH}$, Kamijo $\mathrm{K}$, Scudder M. A review of chronic and acute physical activity participation on neuroelectric measures of brain health and cognition during childhood. Prev Med 2011;52(Suppl 1):S21-8. https://doi.org/10.1016/j.ypmed.2011.01.024

23.Chang YK, Labban JD, Gapin JI, Etnier JL. The effects of acute exercise on cognitive performance: a meta-analysis [published correction appears in Brain Res. 2012 Aug 27;1470:159]. Brain Res 2012;1453:87-101. https://doi.org/10.1016/j.brainres.2012.02.068 24.Basso JC, Suzuki WA. The Effects of Acute Exercise on Mood, Cognition, Neurophysiology, and Neurochemical Pathways: A Review. Brain Plast 2017;2:127-52. https://doi.org/10.3233/BPL-160040 
25.Green BN, Johnson CD, Adams A. Writing narrative literature reviews for peer-reviewed journals: secrets of the trade. J Chiropr Med 2006;5:101-17. https://doi.org/10.1016/S0899-3467(07)60142-6

26. Rampersaud GC. Benefits of breakfast for children and adolescents: update and recommendations for practitioners. Am J Lifestyle Med 2009;3:86-103. https://doi.org/10.1177/1559827608327219

27.Szajewska H, Ruszczynski M. Systematic review demonstrating that breakfast consumption influences body weight outcomes in children and adolescents in Europe. Crit Rev Food Sci Nutr 2010;50:113-9. https://doi.org/10.1080/10408390903467514

28.Barr SI, DiFrancesco L, Fulgoni VL 3rd. Breakfast consumption is positively associated with nutrient adequacy in Canadian children and adolescents [published correction appears in $\mathrm{Br}$ ] Nutr 2015;113:190. $\mathrm{Br}$ J Nutr 2014;112:1373-83.

https://doi.org/10.1017/S0007114514002190

29.Laterra J, Keep R, Betz LA, Goldstein GW. Blood-Brain Barrier. In: Siegel GJ, Agranoff BW, Albers RW (eds.). Basic Neurochemistry: Molecular, Cellular and Medical Aspects. 6th edition. Philadelphia: Lippincott-Raven; 1999.

https://www.ncbi.nlm.nih.gov/books/NBK28180/

30.Paoli A, Tinsley G, Bianco A, Moro T. The Influence of Meal Frequency and Timing on Health in Humans: The Role of Fasting. Nutrients 2019;11:719. https://doi.org/10.3390/nu11040719

31.Mink JW, Blumenschine RJ, Adams DB. Ratio of central nervous system to body metabolism in vertebrates: its constancy and functional basis. Am J Physiol 1981;241:R203-12. https://doi.org/10.1152/ajpregu.1981.241.3.R203

32. Kuzawa CW, Blair C. A hypothesis linking the energy demand of the brain to obesity risk. Proc Natl Acad Sci U S A 2019;116:13266-75. https://doi.org/10.1073/pnas.1816908116

33.Pollitt E, Leibel RL, Greenfield D. Brief fasting, stress, and cognition in children. Am J Clin Nutr 1981;34:1526-33. https://doi.org/10.1093/ajen/34.8.1526

34. Kobayashi F, Ogata H, Omi N, Nagasaka S, Yamaguchi S, Hibi M, et al. Effect of breakfast skipping on diurnal variation of energy metabolism and blood glucose. Obes Res Clin Pract 2014;8:e201-98. https://doi.org/10.1016/j.orcp.2013.01.001

35.Nas A, Mirza N, Hägele F, Kahlhofer J, Keller J, Rising R, et al. Impact of breakfast skipping compared with dinner skipping on regulation of energy balance and metabolic risk. Am J Clin Nutr 2017;105:1351-61. https://doi.org/10.3945/ajcn.116.151332

36.Sanchez-Aguadero N, Garcia-Ortiz L, Patino-Alonso MC, MoraSimon S, Gomez-Marcos MA, Alonso-Dominguez R, et al. Postprandial effect of breakfast glycaemic index on vascular function, glycaemic control and cognitive performance (BGI study): study protocol for a randomised crossover trial. Trials 2016;17:516. https://doi.org/10.1186/s13063-016-1649-x

37.Edefonti V, Rosato V, Parpinel M, Nebbia G, Fiorica L, Fossali E, et al. The effect of breakfast composition and energy contribution on 
cognitive and academic performance: a systematic review. Am J Clin Nutr 2014;100:626-56. https://doi.org/10.3945/ajcn.114.083683

38. Wesnes KA, Pincock C, Scholey A. Breakfast is associated with enhanced cognitive function in schoolchildren. An internet based study. Appetite 2012;59:646-9. https://doi.org/10.1016/j.appet.2012.08.008

39.Brasil. Ministério da Saúde. Secretaria de Atenção à Saúde. Departamento de Atenção Básica. Guia alimentar para a população brasileira (endereço na internet). Brasília: Ministério da Saúde; 2014, $156 \mathrm{p}$.

https://bvsms.saude.gov.br/bvs/publicacoes/guia alimentar populac ao brasileira 2ed.pdf

40.WHO - World Health Organization. Guideline: sugars intake for adults and children (endereço na internet). Acessado em: 04/03/2015. Disponível em: who.int/publications/i/item/9789241549028

41.Gilsenan MB, de Bruin EA, Dye L. The influence of carbohydrate on cognitive performance: a critical evaluation from the perspective of glycaemic load. Br J Nutr 2009;101:941-9. https://doi.org/10.1017/S0007114508199019

42.Foster-Powell $\mathrm{K}$, Holt $\mathrm{SH}$, Brand-Miller JC. International table of glycemic index and glycemic load values: 2002. Am J Clin Nutr 2002;76:5-56. https://doi.org/10.1093/ajen/76.1.5

43.Ingwersen J, Defeyter MA, Kennedy DO, Wesnes KA, Scholey AB. A low glycaemic index breakfast cereal preferentially prevents children's cognitive performance from declining throughout the morning. Appetite https://doi.org/10.1016/j.appet.2006.06.009

44.Cooper SB, Bandelow S, Nute ML, Morris JG, Nevill ME. Breakfast glycaemic index and cognitive function in adolescent school children. $\mathrm{Br}$ J Nutr 2012;107:1823-32.

https://doi.org/10.1017/S0007114511005022

45. Micha R, Rogers PJ, Nelson M. The glycaemic potency of breakfast and cognitive function in school children. Eur J Clin Nutr 2010;64:94857. https://doi.org/10.1038/ejcn.2010.96

46. Micha R, Rogers PJ, Nelson M. Glycaemic index and glycaemic load of breakfast predict cognitive function and mood in school children: a randomised controlled trial. $\mathrm{Br} \mathrm{J}$ Nutr 2011;106:1552-61. https://doi.org/10.1017/S0007114511002303

47.Benton D, Maconie A, Williams C. The influence of the glycaemic load of breakfast on the behaviour of children in school. Physiol Behav 2007;92:717-24. https://doi.org/10.1016/j.physbeh.2007.05.065

48.Brindal E, Baird D, Slater A, Danthiir V, Wilson C, Bowen J, et al. The effect of beverages varying in glycaemic load on postprandial glucose responses, appetite and cognition in 10-12-year-old school children. $\mathrm{Br} \quad \mathrm{J}$ Nutr 2013;110:529-37. https://doi.org/10.1017/S0007114512005296

49.Jennings A, Cassidy A, van Sluijs EM, Griffin SJ, Welch AA. Associations between eating frequency, adiposity, diet, and activity in 
9-10 year old healthy-weight and centrally obese children. Obesity 2012;20:1462-8. https://doi.org/10.1038/oby.2012.72

50.Wagner A, Dallongeville J, Haas B, Ruidavets JB, Amouyel P, Ferrieres J, et al. Sedentary behaviour, physical activity and dietary patterns are independently associated with the metabolic syndrome. Diabetes Metab

https://doi.org/10.1016/j.diabet.2012.04.005

2012;38:428-35.

51. Nicholson JK, Holmes E, Kinross J, Burcelin R, Gibson G, Jia W, et al. Host-gut microbiota metabolic interactions. Science 2012;336:1262-7. https://doi.org/10.1126/science.1223813

52. Rajilić-Stojanović M. Function of the microbiota. Best Pract Res Clin Gastroenterol

2013;27:5-16.

https://doi.org/10.1016/j.bpg.2013.03.006

53. Holscher HD. Dietary fiber and prebiotics and the gastrointestinal microbiota. Gut Microbes 2017;8:172-84.

https://doi.org/10.1080/19490976.2017.1290756

54.Papathanasopoulos A, Camilleri M. Dietary fiber supplements: effects in obesity and metabolic syndrome and relationship to gastrointestinal functions. Gastroenterology 2010;138:65-72. https://doi.org/10.1053/j.gastro.2009.11.045.

55.Sandercock GR, Voss C, Dye L. Associations between habitual school-day breakfast consumption, body mass index, physical activity and cardiorespiratory fitness in English schoolchildren. Eur J Clin Nutr 2010;64:1086-92. https://doi.org/10.1038/ejcn.2010.145

56. Hawley JA, Hargreaves M, Joyner MJ, Zierath JR. Integrative biology of exercise. Cell 2014;159:738-49.

https://doi.org/10.1016/j.cell.2014.10.029

57.Drollette ES, Scudder MR, Raine LB, Moore RD, Saliba BJ, Pontifex $M B$, et al. Acute exercise facilitates brain function and cognition in children who need it most: an ERP study of individual differences in inhibitory control capacity. Dev Cogn Neurosci 2014;7:53-64. https://doi.org/10.1016/j.den.2013.11.001

58.Dias MB, Pereira RMS, Brito SV, Browne R, Ramos IA, Campbell CSG. Efeito de brincadeiras ativas sobre o desempenho escolar em crianças. Edu Fís Rev 2013;7:1-17. https://portalrevistas.ucb.br/index.php/efr/article/view/3938

59.Kamijo K, Pontifex MB, O'Leary KC, Scudder MR, Wu CT, Catelli DM, et al. The effects of an afterschool physical activity program on working memory in preadolescent children. Dev Sci 2011;14:1046-58. https://doi.org/10.1111/j.1467-7687.2011.01054.x

60. Hillman CH, Pontifex MB, Castelli DM, Khan NA, Raine LB, Scudder $M R$, et al. Effects of the FITKids randomized controlled trial on executive control and brain function. Pediatrics 2014;134:e1063-71. https://doi.org/10.1542/peds.2013-3219

61.Donnelly JE, Lambourne K. Classroom-based physical activity, cognition, and academic achievement. Prev Med 2011;52(Suppl 1):S36-42. https://doi.org/10.1016/j.ypmed.2011.01.021

62.Guinhouya BC, Lemdani M, Vilhelm C, Hubert H, Apété GK, Durocher A. How school time physical activity is the "big one" for daily 
activity among schoolchildren: a semi-experimental approach [published correction appears in J Phys Act Health 2009;6:674]; ] Phys Act Health 2009;6:510-9. https://doi.org/10.1123/jpah.6.4.510

63.van den Berg V, Salimi R, de Groot RHM, Jolles J, Chinapaw MJM, Singh AS. "It's a Battle... You Want to Do It, but How Will You Get It Done?": Teachers' and Principals' Perceptions of Implementing Additional Physical activity in School for Academic Performance. Int J Environ Res Public Health 2017;14:1160. https://doi.org/10.3390/ijerph14101160

64.Kohl HW III, Cook HD, Committee on Physical Activity and Physical Education in the School Environment; Food and Nutrition Board; Institute of Medicine (eds.). Educating the Student Body: Taking Physical Activity and Physical Education to School. Washington (DC): National Academies Press (US); 2013. https://doi.org/10.17226/18314

65.Pellegrini AD, Davis PD. Relations between children's playground and classroom behaviour. $\mathrm{Br} \mathrm{J}$ Educ Psychol 1993;63:88-95. https://doi.org/10.1111/j.2044-8279.1993.tb01043.x

66.WHO (World Health Organization). Physical Activity (endereço na internet). Acessado em 23/02/2018. Disponível em: https://www.who.int/en/news-room/fact-sheets/detail/physicalactivity

67. American College of Sports Medicine. ACSM's guidelines for exercise testing and prescription. Philadelphia: Lippincott Williams \& Wilkins, 2014.

68.Jarrett OS, Maxwell DM, Dickerson C, Hoge P, Davies G, Yetley A. Impact of recess on classroom behavior: Group effects and individual differences. J Edu Res 1998;92:121-6. https://doi.org/10.1080/00220679809597584

69. Ramos IA, Costa RC, Brito SV, Aguiar SS, Castro HO, Campbell CSG. Associação entre aptidão física, estratégias de aprendizagem e desempenho escolar em crianças de nove a 11 anos. J Phys Educ 2018;29:e-2973. https://doi.org/10.4025/jphyseduc.v29i1.2973 70. Mahar MT, Murphy SK, Rowe DA, Golden J, Shields AT, Raedeke TD. Effects of a classroom-based program on physical activity and on-task behavior. Med Sci Sports Exerc 2006;38:2086-94. https://doi.org/10.1249/01.mss.0000235359.16685.a3

71. Mahar MT. Impact of short bouts of physical activity on attentionto-task in elementary school children. Prev Med 2011;52(Suppl 1):S60-4. https://doi.org/10.1016/j.ypmed.2011.01.026

72.Egger F, Benzing V, Conzelmann A, Schmidt M. Boost your brain, while having a break! The effects of long-term cognitively engaging physical activity breaks on children's executive functions and academic achievement. PLoS One 2019;14:e0212482. https://doi.org/10.1371/journal.pone.0212482

73. Brito SV, Dias MB, Ramos IAV, Pereira RM, Bodnariuc EF, Campbell CSG. Brincando e aprendendo: Aprimorando o desempenho escolar e comportamento de crianças através de aulas de reforço de educação 
física escolar integradas ao conteúdo escolar. LICERE 2017;20:305-33. https://doi.org/10.35699/1981-3171

74.Ericsson I. Motor skills, attention and academic achievements - an intervention study in school year 1-3. Bri Educ Res J 2008;34:301-13. https://doi.org/10.1080/01411920701609299

75.Grieco LA, Jowers EM, Bartholomew JB. Physically active academic lessons and time on task: the moderating effect of body mass index. Med Sci Sports Exerc 2009;41:1921-6.

https://doi.org/10.1249/MSS.0b013e3181a61495

76.Castelli DM, Glowacki E, Barcelona JM. Active Education: Growing Evidence on Physical Activity and Academic Performance. San Diego: Active Living Research, 2015. https://activelivingresearch.org/sites/activelivingresearch.org/files/AL $\mathrm{R}$ Brief ActiveEducation Jan2015.pdf

77. Diamond A. Close interrelation of motor development and cognitive development and of the cerebellum and prefrontal cortex. Child Dev 2000;71:44-56. https://doi.org/10.1111/1467-8624.00117

78.Davis CL, Tomporowski PD, McDowell JE, Austin BP, Miller PH, Yanasak $\mathrm{NE}$, et al. Exercise improves executive function and achievement and alters brain activation in overweight children: a randomized, controlled trial. Health Psychol 2011;30:91-8. https://doi.org/10.1037/a0021766

79. Howie EK, Schatz J, Pate RR. Acute Effects of Classroom Exercise Breaks on Executive Function and Math Performance: A Dose-Response Study. Res Q Exerc Sport 2015;86:217-24. https://doi.org/10.1080/02701367.2015.1039892

80. Have M, Nielsen JH, Ernst MT, Geji AK, Fredens K, Grontved A, et al. Classroom-based physical activity improves children's math achievement - a randomized controlled trial. PLoS One 2018;13:e0208787. https://doi.org/10.1371/journal.pone.0208787

81.Trudeau F, Shephard RJ. Relationships of Physical Activity to Brain Health and the Academic Performance of Schoolchildren. Am J Lifestyle Med 2010;4:138-50. https://doi.org/10.1177/1559827609351133

82.Chomitz VR, Slining MM, McGowan RJ, Mitchell SE, Dawson GF, Hacker KA. Is there a relationship between physical fitness and academic achievement? Positive results from public school children in the northeastern United States. J Sch Health 2009;79:30-7. https://doi.org/10.1111/j.1746-1561.2008.00371.x

83. Drollette ES, Scudder MR, Raine LB, Moore RD, Saliba BJ, Pontifex $M B$, et al. Acute exercise facilitates brain function and cognition in children who need it most: an ERP study of individual differences in inhibitory control capacity. Dev Cogn Neurosci 2014;7:53-64. https://doi.org/10.1016/j.dcn.2013.11.001

84.Altenburg TM, Chinapaw MJ, Singh AS. Effects of one versus two bouts of moderate intensity physical activity on selective attention during a school morning in Dutch primary schoolchildren: A randomized controlled trial. J Sci Med Sport 2016;19:820-4. https://doi.org/10.1016/j.jsams.2015.12.003 
85.Vazou S, Smiley-Oyen A. Moving and academic learning are not antagonists: acute effects on executive function and enjoyment. J Sport Exerc Psychol 2014;36:474-85. https://doi.org/10.1123/jsep.2014-0035

86.Brocki KC, Bohlin G. Executive functions in children aged 6 to 13: a dimensional and developmental study. Dev Neuropsychol 2004;26:571-93. https://doi.org/10.1207/s15326942dn2602 3

87. Chaddock L, Pontifex MB, Hillman CH, Kramer AF. A review of the relation of aerobic fitness and physical activity to brain structure and function in children. J Int Neuropsychol Soc 2011;17:975-85. https://doi.org/10.1017/S1355617711000567

88.Scudder MR, Drollette ES, Szabo-Reed AN, Lambourne K, Fenton $\mathrm{CI}$, Donnelly $\mathrm{JE}$, et al. Tracking the relationship between children's aerobic fitness and cognitive control. Health Psychol 2016;35:967-78. https://doi.org/10.1037/hea0000343

89. Monti JM, Hillman $\mathrm{CH}$, Cohen NJ. Aerobic fitness enhances relational memory in preadolescent children: the FITKids randomized control trial. Hippocampus 2012;22:1876-82.

https://doi.org/10.1002/hipo.22023

90.Chaddock L, Erickson KI, Prakash RS, VanPatter M, Voss MW, Pontifex MB, et al. Basal ganglia volume is associated with aerobic fitness in preadolescent children. Dev Neurosci 2010;32:249-56. https://doi.org/10.1159/000316648

91.Chaddock L, Erickson KI, Prakash RS, Voss MW, VanPatter M, Pontifex MB, et al. A functional MRI investigation of the association between childhood aerobic fitness and neurocognitive control. Biol Psychol

https://doi.org/10.1016/j.biopsycho.2011.10.017

92.LeBlanc AG, Katzmarzyk PT, Barreira TV, Broyles ST, Chaput JP, Church TS, et al. Correlates of Total Sedentary Time and Screen Time in 9-11 Year-Old Children around the World: The International Study of Childhood Obesity, Lifestyle and the Environment. PLoS One 2015;10:e0129622. https://doi.org/10.1371/journal.pone.0129622

93.Galaviz KI, Zytnick D, Kegler MC, Cunningham SA. Parental Perception of Neighborhood Safety and Children's Physical Activity. J Phys Act Health 2016;13:1110-6. https://doi.org/10.1123/jpah.20150557

94.Molnar BE, Gortmaker SL, Bull FC, Buka SL. Unsafe to play? Neighborhood disorder and lack of safety predict reduced physical activity among urban children and adolescents. Am J Health Promot 2004;18:378-86. https://doi.org/10.4278/0890-1171-18.5.378

95.Núcleo de Informação e Coordenação do Ponto BR (eds). Pesquisa sobre o uso da internet por crianças e adolescentes no Brasil: TIC Kids online Brasil 2015. São Paulo: Comitê Gestor da Internet no Brasil, 2016.

https://cetic.br/media/docs/publicacoes/2/TIC Kids 2015 LIVRO EL ETRONICO.pdf

96.Telama R, Yang X, Viikari J, Välimäki I, Wanne O, Raitakari O. Physical activity from childhood to adulthood: a 21-year tracking study. 
Am J Prev Med 2005;28:267-73.

https://doi.org/10.1016/j.amepre.2004.12.003

97.Movassagh EZ, Baxter-Jones ADG, Kontulainen S, Whiting SJ, Vatanparast $\mathrm{H}$. Tracking Dietary Patterns over 20 Years from Childhood through Adolescence into Young Adulthood: The Saskatchewan Pediatric Bone Mineral Accrual Study. Nutrients 2017;9:990. https://doi.org/10.3390/nu9090990 